\title{
New calibration of partial material factors for the structural design of float glass. Comparison of bounded and unbounded statistics for glass strength
}

\author{
Roberto Ballarini \\ Department of Civil and Environmental Engineering \\ Cullen College of Engineering, University of Houston, TX, USA \\ Gabriele Pisano \\ Department of Industrial Engineering, University of Parma \\ Parco Area delle Scienze 181/A, I 43100 Parma, Italy \\ Gianni Royer Carfagni \\ Department of Industrial Engineering, University of Parma \\ Parco Area delle Scienze 181/A, I 43100 Parma, Italy
}

\begin{abstract}
Partial material factors need to be calibrated to guarantee the target failure probability of glass structures according to the semi-probabilistic (level I) methods of design. Calibration is made by comparison with results obtainable with the full probabilistic approach (level III) on paradigmatic case studies. Considering the results of previous work that assessed the validity of various statistical models of glass strength, we use a generalized distribution of the Weibull type to derive new partial factors that account for a lower bound for glass strength or not. The partial factors so calculated are much lower than those previously obtained from the classical two-parameter Weibull distribution, and are in agreement with the coefficients commonly used in practice. Moreover, the variation of the partial factors with respect to changes in failure probability is similar to what is applied to other building materials.
\end{abstract}

Email addresses: rballarini@uh.edu (Roberto Ballarini), gabriele.pisano@studenti.unipr.it (Gabriele Pisano), gianni.royer@unipr.it (Gianni Royer Carfagni )

Preprint submitted to Elsevier

May 20, 2016

(C) 2016. This manuscript version is made available under the Elsevier user license http://www.elsevier.com/open-access/userlicense/1.0/ 
Keywords: Glass, float glass, probabilistic design, semi-probabilistic approach, partial safety factors, statistical distribution, calibration, Weibull distribution.

\section{Introduction}

Glass is a very old material whose use in construction works has radically changed over the past few decades. It has evolved from a simple in-fill material for windows to an effective structural material of ever-increasing use to build roofs, floors, balustrades and stairs. There is the need to develop specific design methods to assure for glass structures safety levels comparable with those usually required for more traditional civil engineering construction works. Structural performance is typically defined by the maximum acceptable probability of collapse, as assigned by the relevant structural codes. In order to guarantee such performance, the semiprobabilistic method of design (level I) is usually employed: partial amplifying factors for the actions and partial reduction factors for the resistances are used, so as to ensure that the probability of failure is equal or lower than the target value. The partial factors for actions and materials are prescribed by structural codes on the basis of calibration from a full probabilistic approach and from practical experience. However, what are the correct material factors for glass is a matter of debate.

Glass presents many peculiarities with respect to other building materials. First of all, it is brittle. Its macroscopic strength is determined by the opening of small flaws on its surface whose shape is usually approximated as "thumbnail." Because there is no possibility of stress redistribution through plasticity-type phenomena, catastrophic failure of the whole

glass structural component occurs when the opening mode Stress Intensity Factor (SIF) of the dominant crack reaches a critical value defined as the fracture toughness. However, it has been recognized that the cracks could extend even when the SIF is smaller than this critical limit, due to a phenomenon [1] referred to as subcritical crack propagation, or static fatigue. The susceptibility of glass to static fatigue must be taken into account in the design. The critical role played by a single crack is the reason why Weibull's weakest-link-in-the-chain 
concept [2] is universally accepted to interpret the variability of the measured strength of glass.

The strong dependence of the macroscopic response of glass on the existence of small flaws requires the consideration of aspects that are not as important for other building materials. One of these is structural size; the larger the loaded surface, the higher is the probability of finding a flaw associated with the critical combination of size and stress. The probability of failure is also dependent on the distribution of stress. For example, if the stress is uniform equi-biaxial the orientation of the cracks does not matter, but if the stress is uniaxial there is a lower probability that the surfaces of the dominant crack are perpendicular to the direction of the maximal tensile stress. And finally, the strength of glass is highly dependent on changes in the flaw population produced by the manufacturing process and subsequent handling. In the float production process, patented in the late 1950s by Sir Alastair Pilkington, a glass paste is poured on a bed of molten tin so to form a floating panel. The contact of the panel with tin, and with the successive contact with the rollers, can induce a higher level of surface damage on the "tin side" with respect to the "air-side". The cutting process may also cause additional damage and hence a local reduction of strength at the borders. All these aspects must be considered in the assessment of the expected probability of failure.

The failure probability that is considered acceptable depends on the consequence of the potential collapse and is prescribed by structural standards. This classification, indicated by the standard for the entire structure, should be detailed to the single structural element on the basis of the consequences of its own collapse. In Europe, the EN 1990 [3] indicates the accepted probability of failure for three Classes of Consequences (CC), varying from $1.33510^{-5}$ for $\mathrm{CC} 1$, to $1.30510^{-6}$ for $\mathrm{CC} 2$ and to $9.9610^{-8}$ (CC3) in one year. By considering that the actions are the same for all structures, the required probability scenario is defined on basis of the glass resistance and the partial material factors $\gamma_{m}$. The complete probabilistic method (level III) directly measures the failure probability through the convolution of the cumulative probability for glass strength and the probability density function for the actions, and it is generally used for calibrating the $\gamma_{m}$. This is why the statistical model of material 
strength very strongly affects the values for the material partial factors. In particular, when such low failure probabilities are considered, a very accurate characterization of the left-handside tail of the population of the material strength becomes of primary importance.

The strength of glass is usually modeled using the traditionall two-parameter Weibull (2PW) extreme value distribution. However, it cannot provide [4] an accurate description on the left-hand-side tail. Moreover, there are reasons in support of the existence of an intrinsic lower bound for the strength of commercial float glass [5]. A minimum strength value is attributed to rigorous factory production controls of visual transparency that concomitantly guarantee that the sizes of the surface defects remain well below an assigned value. While considering the capability of various types of generalized Weibull distributions in interpreting the experimental data, it has been shown [5], using the goodness of fit procedure, that "bounded" Weibull statistics, i.e., statistics assuming a lower-bound for population, are more accurate than "unbounded" statistics (especially for the air-side). One may be concerned with potential strength degradation from aging. It is true that natural abrasion/corrosion of the glass surfaces can increase the surface damage in glass. However there is substantial evidence that even the worst deterioration produced by sand blasting cannot push the strength of glass below a certain limit [5].

To our knowledge, all of the proposed design methods are based on the two-parameter Weibull distribution. Using this statistics, the probabilistic method of level III was applied for the verification of paradigmatic case studies [6], which served to calibrate the partial material factors $\gamma_{m}$ to be used in the semi-probabilistic approach of level I. To distinguish the different classes of consequences, each one characterized by the target probability of collapse assigned by standard EN 1990 [3], a multiplication coefficient for the partial material factor was introduced instead of that for the partial factors of loads. However, the values of the $\gamma_{m}$ so obtained are quite high, of the order of 2.55 for CC2 and 1.8 for CC1. In fact the values for CC3 were not even recorded because they were considered to be so high that they deserve further investigation. Note that the values were calibrated for small-size plates (of the order of $1 \mathrm{~m}^{2}$ ), so that larger structures will be associated with even higher 
values. The values required by the two-parameter Weibull models, being so much higher than those traditionally used for the design of glass elements (which are based upon practical rules, construction tradition and professional experience), have been hardly accepted by the building industry.

This paper presents a new calibration of partial factors that relies on improved statistical distributions of glass strength. With respect to the paper by Badalassi et al. [6], the considered population of glass strengths derives from a much wider experimental campaign [7], obtained with a refined testing method [8]. The statistical model considered here is the "bounded" left-truncated Weibull (LTW) distribution that provides an excellent goodness of fit with experimental data, at least on the air side [5]. Because a lower limit for glass strength may be hard to accept, a comparison is made with the "unbounded" extended

Weibull (EXW) distribution that, although not optimal [5], gives much better results than the 2PW. Both the LTW and EXW distributions are derived by the $2 \mathrm{PW}$ distribution and provide similar rescaling of glass strength to account for the effects of size and type of stress, with no major analytical complications. The new coefficients calibrated with the LTW distribution are substantially lower than those presented by Badalassi et al. [6] and are in agreement with the indications of practical experience. Moreover, the variation of the coefficients to pass from one class of consequences to another is much more limited and of the same order of magnitude of what is suggested in the EN 1990 [3] for other building materials. The results from the EXW distribution is intermediate between these and the calibration obtained with the $2 \mathrm{PW}$ statistics.

\section{The statistical modelling of glass strength}

We first provide a brief review of the experimental campaign used as the reference for the derivation of generalized Weibull distributions, capable of interpreting the left-hand-side tail of the population (at very low failure probabilities) better than the $2 \mathrm{PW}$ model, in addition to a discussion of possible effects on glass strength of aging due to natural abrasion/corrosion. 


\subsection{Experimental data}

The experimental data used to calibrate the statistical parameters are those from the experimental campaign by the working group TC129/WG8 of CEN that, to our knowledge, is the widest ever made for float glass. The measured strengths from about 740 coaxial-double-ring tests with additional overpressure, performed according to the standard EN1288-2 [8], are reported in a technical document by CEN/TC129/WG8 [7]. It has been explained [4] that such data required corrections and re-scaling to compensate for the rough approximations made in the original interpretation and elaboration. Moreover, the failure stress values are representative of the instantaneous collapse of the specimen [4], thus they neglect the effects of static fatigue recalled in Appendix A. This is reasonable because the time to failure during the experiments is of the order of seconds and the approximation is on the safe side, since the instantaneous strength is certainly higher than the delayed strength for static fatigue.

For what follows a distinction must be made between the population of strengths measured either on the tin or on the air side of float glass. This because the corresponding statistics are characterized by considerably different parameters that result from the float production process; the face of glass merged in the tin bath, and successively in contact with the roller supports, is characterized by a significantly higher level of surface damage than the face exposed to air.

\subsection{Unbounded Weibull distributions}

We refer to a Weibull distribution as "unbounded" if the domain for the failure stress is $[0,+\infty)$.

In the two-parameter Weibull (2PW), the probability of failure of an element of surface area $A$, under a generic tensile state of stress, reads

$$
P_{f, W 2}=1-\exp \left[-\int_{A}\left(\frac{\sigma_{e q, W 2}}{\eta_{0}}\right)^{m} d A\right]=1-\exp \left[-K_{2 W} A\left(\frac{\sigma_{\max }}{\eta_{0}}\right)^{m}\right]
$$


where $m$ and $\eta_{0}$ are the shape and the scale parameters, respectively, $\sigma_{e q, W 2}$ represents an equivalent stress field accounting for the distribution of tensile stresses, $\sigma_{\max }$ is the maximum tensile stress on the stressed surface and $K_{2 W} A$ is the corresponding effective area.

Let $\sigma_{1}$ and $\sigma_{2}\left(\sigma_{1} \geq \sigma_{2}\right)$ be the principal components of the tensile stress and let $\psi$ be the angle between the direction of $\sigma_{1}$ and the normal to surfaces of the dominant crack. Assuming an isotropic orientation of defects, one can thus define

$$
\sigma_{e q, W 2}=\left[2 / \pi \int_{0}^{\pi / 2}\left(\sigma_{1} \cos ^{2} \psi+\sigma_{2} \sin ^{2} \psi\right)^{m} d \psi\right]^{1 / m}
$$

and, from the condition of equal failure probability, the effective area $K_{2 W} A$ reads

$$
K_{W 2} A=A_{e f, W 2}=\frac{\int_{A}\left(\sigma_{e q, W 2}\right)^{m} d A}{\left(\sigma_{\max }\right)^{m}} .
$$

In order to give a better interpretation of the experimental data, Marshall and Olkin [9] proposed a method for adding a parameter to the two-parameter Weibull distribution. The extended Weibull (EXW) distribution is of the form

$$
P_{f, W E}=1-\frac{\nu \widetilde{P}_{s}}{1-\left(1-\nu \widetilde{P}_{s}\right)}
$$

where $\widetilde{P}_{s}$ is a two parameter distribution, whose expression is of the same type as (2.1), and $\nu$ is the third parameter to be calibrated. Clearly the equivalent stress $\sigma_{e q, W E}$ and the effective area $A_{e f, W E}$ assume forms identical to those of (2.2) and (2.3), respectively.

Among all the unbounded Weibull distributions considered in previous papers [4, 5], the EXW distribution appears to be the most convenient to interpret the experimental data. Other analyzed models, including the bilinear and the bimodal distributions, not only have very limited accuracy but also present formidable analytical difficulties in their definition of the effective area. This renders their practical use problematic at best. 
It should be mentioned that some authors [10] have proposed, outside the class of statistics of the Weibull type, the use of the lognormal distribution ${ }^{1}$. It was shown that this statistical model gives a fair goodness of fit [4] for the data recorded in the technical document by CEN/TC129/WG8 [7]. However it is not a robust model for glass strength because it does not explicitly consider the effects of size and stress state.

\subsection{Bounded Weibull distibutions}

The "bounded" Weibull distributions include a lower bound $\sigma_{0}>0$ for glass strength and, hence, the domain for the failure stress is $\left[\sigma_{0},+\infty\right)$.

For the 3-parameter Weibull (3PW) distribution, the cumulative failure probability reads

$$
P_{f, W 3}=1-\exp \left[-\int_{A}\left(\frac{\sigma_{e q, W 3}-\sigma_{0}}{\eta_{0}}\right)^{m} d A\right]=1-\exp \left[-K_{W 3} A\left(\frac{\sigma_{\max }-\sigma_{0}}{\eta_{0}}\right)^{m}\right]
$$

where $m, \eta_{0}$ and $\sigma_{0}$ are the shape, scale and location parameters, respectively. With the same notation of (2.2), the equivalent stress field $\sigma_{e q, W 3}$ now takes the form

$$
\sigma_{e q, W 3}-\sigma_{0}=\frac{2}{\pi} \int_{0}^{\pi / 2}\left(\left|\sigma_{1}-\sigma_{0}\right|^{+} \cos ^{2} \psi+\left|\sigma_{2}-\sigma_{0}\right|^{+} \sin ^{2} \psi\right) d \psi
$$

and the corresponding effective area $K_{3 W} A$ is

$$
A_{e f, W 3}=K_{W 3} A=\frac{\int_{A}\left(\sigma_{e q, W 3}-\sigma_{0}\right)^{m} d A}{\left(\sigma_{\max }-\sigma_{0}\right)^{m}}
$$

\footnotetext{
${ }^{1}$ In the lognormal statistics, the logarithm of the random variable $x$ is normally distributed. The density function $f(x)$ reads

$$
f(x)=\frac{1}{x \sqrt{2 \pi \zeta^{2}}} \exp \left[-\frac{1}{2} \frac{(\log (x)-\mu)^{2}}{\zeta^{2}}\right], \quad 0<x<+\infty,
$$

where $\mu$ and $\zeta^{2}$ are the mean and the variance of the logarithm of the data, respectively, which consequently do not represent the expectation value and the variance of the stochastic variable.
} 
The presence of $\sigma_{0}$ in equations (2.7) and (2.8) leads to a strong nonlinear dependence of the effective area upon the state of stress. This fact makes it very difficult to apply this statistical model. This complication does not appear in an expression of the type (2.3).

A very accurate goodness of fit with the experimental data can also be achieved with a left-truncated Weibull (LTW) distribution [5]. This is the distribution that is obtained from the population obeying to a $2 \mathrm{PW}$ distribution when all the specimens with strength lower than a certain limit $\sigma_{0}$ are not accepted. This would apply when ideal factory production controls mitigate the possibility of levels of surface damage higher than a specified value. The cumulative probability of failure is of the form

$$
P_{f, W T}=1-\exp \left[-\frac{\int_{A}\left(\sigma_{e q, W T}^{m}-\sigma_{0}^{m}\right) d A}{\eta_{0}^{m}}\right]=1-\exp \left[-\frac{K_{W T} \sigma_{\max }^{m}-\sigma_{0}^{m}}{\eta_{0}^{m}} A\right]
$$

Remarkably, the expression of the equivalent stress field $\sigma_{e q, W T}$ is identical to that of (2.2), and consequently the effective effective area $K_{W T} A$ is analogous to (2.3). This represents the major advantage of the truncated Weibull distribution, i.e., it takes into account a lower bound for glass strength and at the same time it includes a particularly simple re-scaling that could account for the effects of size and stress state.

\subsection{Effects of duration of applied loads and aging}

As mentioned previously, cracks in glass can grow in time when their opening stress is far below the critical limit [1]. A review of static fatigue or subcritical crack growth is provided in Appendix A. In general, applied actions are schematized by loads assumed to remain constant for a characteristic time, representative of their effects during the life-time of the structural element. In design practice, one accounts for the effects of static fatigue by reducing the characteristic design strength of glass through the coefficient $k_{m o d}$, defined in (A.5).

Particular considerations are needed in the case of bounded Weibull statistics. To illustrate, it is convenient to refer to the physical derivation of the LTW distribution, according to 
which the location parameter $\sigma_{0}$ comes from a left truncation of the experimental data. Since the inferred lower bound for glass strength is associated with the maximum size of the surface flaws that can grow in time under subcritical conditions, $\sigma_{0}$ should be re-scaled when taking into account different load histories. In fact, a precise evaluation of $\sigma_{0}$ is crucial for the calibration of partial safety factors, because small changes in its value can lead to noteworthy differences in the interpretation of the left-hand-side tails of the population.

To take into account the effects of static fatigue, once the population of experimental data recorded in the technical document by CEN/TC129/WG8 [7] has been assigned, we suggest to rescale such data using equation (A.4) of Appendix A to obtain a new population, which represents the measured strength for loads that are maintained constant during the characteristic time $\tau$. These data can thus be interpolated again with a new statistical distribution, to obtain the values $\sigma_{0, \tau}$ and $\eta_{0, \tau}$ of the location and scale parameters, respectively. In this process the shape parameter $m$ is assumed to remain uneffected.

It is likewise important to consider the effects of aging, in particular those due to superficial abrasion-corrosion. This phenomenon has been discussed at length in Section 2.3 of the paper [5] for the damage produced by sandblasting, for which two major conclusions were drawn: i) abrasion can increase the stress intensity factor associated with a dominant crack, but $i i$ ) there is an upper bound for this increment because too many defects may produce beneficial shielding (reduction in SIF) of the dominant flaw. Therefore, our suggestion is to operate a further reduction of the location parameter and consider in the statistical distributions the quantity $\sigma_{0, \tau}^{*}$, defined as

$$
\sigma_{0, \tau}^{*}=\frac{\sigma_{0, \tau}}{\omega}
$$

with $\omega>1$. To take into account that all of the strength data, and not only the lower bound $\sigma_{0, \tau}$, are affected by a similar reduction, we also suggest to vary in a similar manner the parameter $\eta_{0}$ in $(2.9)$ by $\eta_{0, \tau}^{*}$ as $p e r$ 


$$
\eta_{0, \tau}^{*}=\frac{\eta_{0, \tau}}{\omega}
$$

As it will made clear in Section 3.3, while discussing Figure 1, such a choice causes a uniform shift on the left-hand-side of the cumulative probability of failure.

A final consideration regards the difference between the tin and air side of float glass. The experimentally-measured difference on the two strength populations should be attributed to a different defectiveness on the two surfaces [5]. It is difficult to estimate precisely such

a difference, but certainly the values of the coefficients $\omega$ of (2.10) and (2.11) should be somehow related for the tin and air side. Albeit tentatively, we propose to consider

$$
\omega_{\text {tin }}=\omega_{\text {air }}(1-d)=\omega(1-d) \Rightarrow \sigma_{0, \tau, t i n}^{*}=\frac{\sigma_{0, \tau, t i n}}{(1-d) \omega}, \sigma_{0, \tau, a i r}^{*}=\frac{\sigma_{0, \tau, a i r}}{\omega}
$$

where $d$ takes into account that the tin side surface is already damaged. Analogous formulas hold for $\eta_{0, \tau, a i r}^{*}$ and $\eta_{0, \tau, t i n}^{*}$. Recalling the results of the statistical analysis of a previous study [4] and, in particular, the fact the ratio between the lower limits for the air and the tin side is $36 / 39 \simeq 0.92$, we propose to take $d=0.10$.

The evaluation of $\omega$ is not straightforward and would require an accurate experimental investigation that considers various possible damaging actions, including those caused by handling. One can expect a reduction of the strength of the order of $10 \div 20 \%$ from the effects of abrasion due to sandblasting, which represents a very severe damaging action $[11,5]$. However, since our aim is the calibration of the partial material factors for structural design, we will choose here the rather conservative value $\omega=1.5$.

\section{The probabilistic and semi-probabilistic approach}

The procedure that will be used in the sequel for the calibration of material partial safety factors is now discussed in detail. The method is based upon a comparison of the results that can be obtained, on paradigmatic case studies, by using the full probabilistic approach of 
level III and the semiprobabilistic approach of level I. The statistical distributions for glass strength that will be considered are the EXW and the LTW distributions that have proved to be the best [5]. Results will be compared with those from the $2 \mathrm{PW}$ distribution obtained by Badalassi et al. [6].

\subsection{Expected performance of glass structures and case studies}

In order to guarantee a certain level of safety against failure, the modern approach to structural design is based upon probabilistic considerations. Structural standards prescribe the probability of collapse that is reputed acceptable, as a function of the consequence of the collapse itself. In Europe, the principles and requirements for the structural safety of buildings and other civil works are established by the Eurocode EN 1990 "Basis of structural design" [3], which is used in conjunction with the other nine standards EN 1991-1999. None of these documents however spell out an approach that should be followed during the design of load-bearing glass components.

The standard EN 1990 defines three classes of consequences, namely CC1, CC2 and CC3, by considering the consequences of failure or malfunction of the construction in economic, social, and environmental terms. Obviously, the choice of the specific class is related with the importance of the construction itself. For example, CC3 refers to grandstands and public buildings, CC2 to residential and office buildings and CC1 to agricultural buildings and greenhouses. For the case of glass components the distinction cannot be made on the basis of the type of construction in within which they are incorporated. On the one hand, it would be rare to find an expensive glass floor in a cowshed: the cost of a glass structure can only be afforded in luxury constructions. On the other hand, assigning to all the glass elements the higher class CC3 would be strongly uneconomical and unjustified, because glass is employed to make only isolated parts of the construction (facades, floors, stairs), whose failure is very rarely accompanied by the collapse of the whole building. However, there are cases for which breakage of one glass element may have comparable consequences, and this is why it is necessary to make a classification on the basis of the single element, rather than of the 
whole construction work. This is the approach that has been followed, for example, by the Italian code for structural glass [12], which has proposed the schematic reported in Table 1. This includes, for completeness, also the class CCO referring to clearly-non-structural elements (for example, a glass pane for a common window).

Table 1: Classes of consequences for glass elements indicate by CNR-DT210 [12].

\begin{tabular}{cl}
\hline Class & \multicolumn{1}{c}{ Definition } \\
\hline CC0 & $\begin{array}{l}\text { Specifically non-structural elements. Following failure, negligible economic, social and environmental consequences } \\
\text { and practically null risk of loss of human life. }\end{array}$ \\
CC1 & $\begin{array}{l}\text { Following failure, low risk of loss of human life and modest or negligible economic, social and environmental } \\
\text { consequences. Glass structural elements whose failure involves scarce consequences fall into to this category. }\end{array}$ \\
CC2 & $\begin{array}{l}\text { Following failure, moderate risk of loss of human life, considerable economic, social and environmental } \\
\text { consequences. Glass structural elements whose failure involves medium-level consequences belong to this category. }\end{array}$ \\
CC3 & $\begin{array}{l}\text { High risk of loss of human life, serious economic, social and environmental consequences: for instance, } \\
\text { the structures of public buildings, stages and covered galleries, where the consequences of failure can be } \\
\text { catastrophic (concert halls, crowded commercial centers, etc.). Glass structural elements whose failure involves } \\
\text { high-level consequences fall into this category }\end{array}$ \\
\hline
\end{tabular}

Concerning the class $\mathrm{CC} 0$, there is not an unanimous agreement about the level of performance to be expected. According to some European standards and guidelines, the assessment of the safety level should be directly obtained from experimental testing of prototypes. The American ASTM 1300 [13], appendix X3.1 presents formulas for estimating the probability of breakage of rectangular glass elements under specific loads, which is stated to be less than 0.05, even if no explicit reference is made whether this value refers to the entire lifetime of the structure. Therefore, this case will be not treated here.

For the other three classes, in Europe the EN 1990 [3] establishes the reference life times for different types of civil structures, defined as the period during which the deterioration does not impair the performances of the structure and only ordinary maintenance is required. Once this is established, the probabilities of collapse can be assigned. Table 2 reports the probabilities of failure for a reference life time of 50 years, given for two different values (1 year and 50 years) of the return period $T_{R}$ of the applied actions. Decreasing probabilities of failure, assumed equal to those computable by using the target values of the corresponding reliability index $\beta$ (also reported in Table 2), can be accepted while passing from the first to 
the third class of consequence.

Table 2: Probability of collapse as a function of the different classes of structural elements according to EN 1990 [3].

\begin{tabular}{ccccc}
\hline Class & $\beta\left[T_{R}=50\right.$ years $]$ & $P_{f}\left[T_{R}=50\right.$ years $]$ & $\beta\left[T_{R}=1\right.$ year $]$ & $P_{f}\left[T_{R}=1\right.$ year $]$ \\
\hline $\mathrm{CC} 1$ & 3.3 & $4.8310^{-4}$ & 4.2 & $1.33510^{-5}$ \\
$\mathrm{CC} 2$ & 3.8 & $7.23510^{-5}$ & 4.7 & $1.30510^{-6}$ \\
$\mathrm{CC} 3$ & 4.3 & $8.5410^{-6}$ & 5.2 & $9.9610^{-8}$ \\
\hline
\end{tabular}

\subsection{Safety factors and the coefficient $R_{M}$}

It is customary [3] to classify the probabilistic calibration procedures for partial factors into two main classes: full probabilistic method (level III) and first order reliability method (FORM - Level II). The Level III methods may be considered the most accurate because they require a direct measurement of the probability of collapse from the statistical distributions of the actions and of the resistances. Denote with $\mathrm{S}$ the domain for actions and with $f_{s}$ the statistical distribution of the values $s \in S$ and, by analogy, with $\mathrm{R}$ the domain of the resistances and with $f_{r}$ the statistical distribution of the values $r \in R$. If actions and resistances are independent variables, the probability of failure $P_{f}$ can be calculated from

$$
P_{f}=P[R-S \leq 0]=\int_{-\infty}^{+\infty} \int_{-\infty}^{s \geq r} f_{R}(r) f_{S}(s) d r d s
$$

When the domains of resistance and action coincide, i.e., $r=s=x, x \in X$, one can write

$$
P_{f}=P[R-S \leq 0]=\int_{-\infty}^{+\infty} F_{R}(x) f_{S}(x) d x,
$$

where $F_{R}(x)$ represent the cumulative distribution of strength, i.e., the probability that the strength is less that $x$. The level II methods provide some hypothesis which simplify the 
estimation of the probability of failure, so that it can be measured by the reliability index $\beta$. In general, these methods are proposed under the assumption that the material strength is interpreted by a Gaussian distribution, which is not applicable to glass.

The material partial safety factors are calibrated by referring to paradigmatic case studies. For these, an optimal design is obtained that achieves the target probability of failure established in Table 2, calculated with level III methods. Then, one finds the value of the material partial safety factor $\gamma_{m}$ that reproduce such optimal design with level I methods, assuming the partial coefficients for actions $\gamma_{f}$ indicated by the relevant standards.

In general, partial safety factors are provided that refer to the probability of failure associated with CC2. To pass to CC1 or CC3, the standard EN 1990 [3] introduces a multiplicative correction factor $K_{F I}$ for the partial coefficient of the actions $\gamma_{f}$. Obviously $K_{F I}<1$ when passing from $\mathrm{CC} 2$ to $\mathrm{CC} 1, K_{F I}>1$ when going from CC2 to CC3. For the case of glass structures, as indicated in CNR-DT 210 [12], it is usually preferred to introduce the correction coefficient $R_{M}$ of the material partial safety factor, so that $R_{M}<(>) 1$ when passing from $\mathrm{CC} 2$ to $\mathrm{CC} 1(\mathrm{CC} 3)$. If the structure is linear elastic, there is no difference between decreasing the actions or increasing the resistances. But in the presence of geometric non-linearities that may be involved in slender glass structures, it seems more appropriate to increase the resistances because otherwise the non-linear effects could be underestimated.

The paradigmatic case studies considered here are associated with a 8mm-thick square glass plate of side $3000 \mathrm{~mm}$, simply supported at the edges under a uniformly distributed outof-plane load. Such load will be representative of the effects of wind on a façade panel, or of the effects of snow on a roof panel. The various loads differ in their characteristic duration. For the wind actions, two different conditions are considered, each one associated with characteristic wind pressures averaged over the times either $t=10$ min or $t=3$ sec. Although the former load value is lower than the latter, its duration is higher and therefore the phenomena of static fatigue, referred to in Appendix A, may render the first verification more restrictive than the second one. Following the recommendations by standards [12], the characteristic values of the snow load will considered applied for a time equal to one month. 
These conditions will be examined in detail in Section 4 .

\subsection{Calibration procedure based upon the left-truncated Weibull (LTW) statistics}

The left-truncated Weibull distribution is represented by (2.9). In order to take into account the effects of static fatigue, the experimentally measured points in the experimental campaign of subsection 2.1 were re-scaled according to the "subcritical crack growth" law of (A.4) according to the characteristic duration $\tau$ of the applied loads, i.e., $3 \mathrm{sec}$ and $10 \mathrm{~min}$, for the two characteristic values of wind load, and one month for the snow load. The statistical distribution representing the material strength for each case study is hence dependent upon $\tau$.

All the parameters that characterize the statistics of glass strength, for either the air or the tin side, are recorded in table 3 as graphically estimated [5]. Recall that the experimental data need to be re-scaled according to the effective area $K_{W T} A$ corresponding to the specimen in the testing configuration of EN 1288-2 [8]. Such an expression is analogous to $K_{W 2} A$ of equation (2.3) and it is stress-dependent if one takes into account geometric non-linearities [14]. However, since the non-linear distribution of stress in the plate is such that the higher the loads, the lower is $K_{W T}$ (conservative), we therefore assumed the values of $K_{W T, a i r}$ and $K_{W T, t i n}$ that correspond to a maximum stress of $100 \mathrm{MPa}$, which is approximately equal to the maximum stress measured in the tests. The values of $\sigma_{0, \tau}^{*}$ and $\eta_{0, \tau}^{*}$ have been calculated as per (2.10) and (2.11).

Figure 1 shows that the reduction of the scale parameter $\eta_{0, \tau}$ and of the location parameter $\sigma_{0, \tau}$ according to (2.10) and (2.11) causes a leftward shift of the cumulative probability function curve and an increase in the slope of the same curve, which means a reduction of the material strength and of the data dispersion.

Since the orientation of the glass plate is random, following Badalassi et al. [6] we will consider that the probability that the air side is under maximum tensile stress is equal to the probability of having the tin side in this condition. Hence, the corresponding cumulative 
Table 3: Graphically estimated parameters characterizing the left-truncated Weibull distribution for wind (two values) and snow loads. For the test configuration of EN 1288-2 [8]: $K_{W T, a i r}=0.54 ; K_{W T, t i n}=$ $0.55 ; G_{\text {air }}=0.065 ; G_{t i n}=0.24 ; A=0.2826 \mathrm{~m}^{2}$ as per [5].

\begin{tabular}{cccccc}
\hline Case study & $m$ & $\begin{array}{c}\eta_{0, \tau} \\
{[\mathrm{MPa}]}\end{array}$ & $\begin{array}{c}\sigma_{0, \tau} \\
{[\mathrm{MPa}]}\end{array}$ & $\begin{array}{c}\eta_{0, \tau}^{*} \\
{[\mathrm{MPa}]}\end{array}$ & $\begin{array}{c}\sigma_{0, \tau}^{*} \\
{[\mathrm{MPa}]}\end{array}$ \\
\hline AIR SIDE & & & & & \\
Wind, 10 min & 4.0025 & 40.12 & 27.27 & 26.75 & 18.53 \\
Wind, 3 sec & 4.0025 & 55.51 & 38.45 & 37.00 & 25.63 \\
Snow, 1 m & 4.0025 & 24.39 & 16.89 & 16.26 & 11.26 \\
TIN SIDE & & & & & \\
Wind, 10 min & 3.7678 & 26.41 & 25.29 & 19.56 & 18.73 \\
Wind, 3 sec & 3.7678 & 36.53 & 34.98 & 27.06 & 25.91 \\
Snow, 1 m & 3.7678 & 24.39 & 16.89 & 16.26 & 11.26 \\
\hline
\end{tabular}

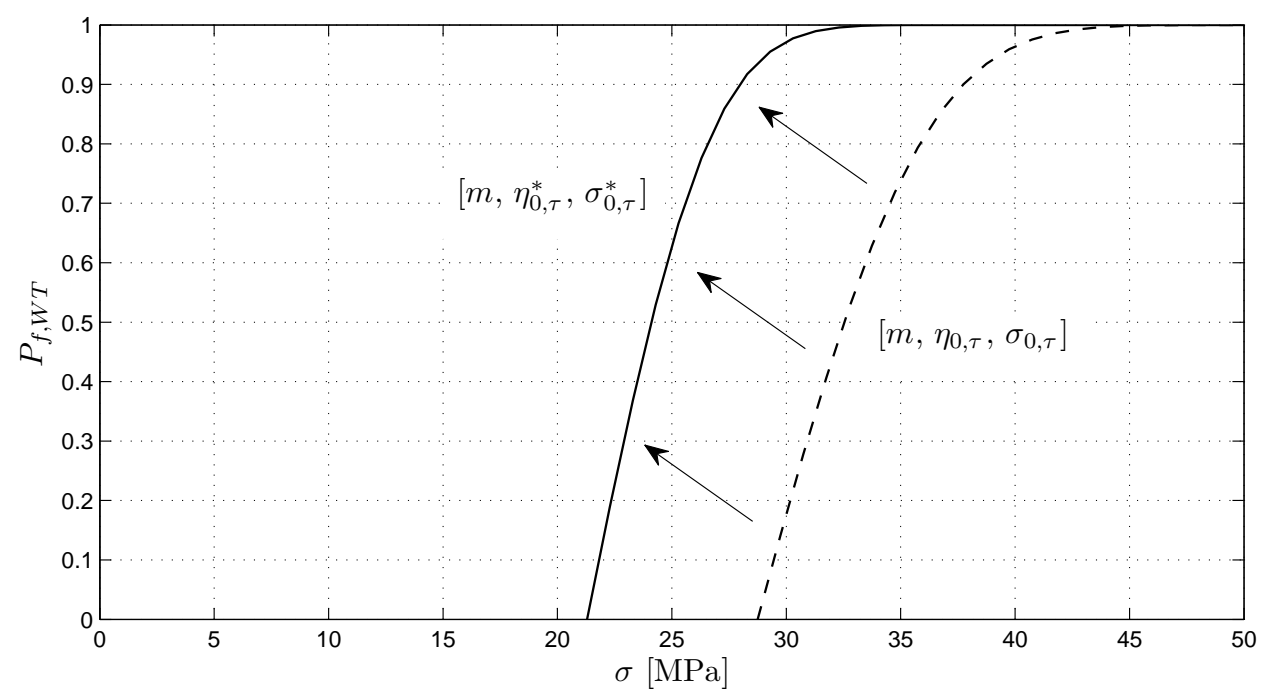

Figure 1: Cumulative probability distribution of the material strength modified for taking into account the effects of aging. Case of $10 \mathrm{~min}$ averaged peak-pressure acting on the air side surface.

probability function of material strengths is the arithmetic mean of the functions representing the strength of the two surfaces. Let symbolically "sd=air" or "sd=tin" represent the 
side "sd" of glass. The cumulative distribution reads

$$
F_{\sigma, A, \tau, W T}^{(a i r+t i n) / 2}=1-\frac{1}{2} \sum_{s d=a i r}^{t i n} \exp \left[-\frac{K_{W T}^{s d} \sigma^{m_{s d}}-\sigma_{0, \tau, s d}^{m_{s d}}}{\eta_{0, \tau, s d}^{m_{s d}}} A\right]
$$

Setting $f_{\sigma, \tau}(\sigma)$ as the distribution probability for the maximum stress $\sigma$ occurring in one year, the convolution integral (3.4), which reads

$$
P_{f, 1 y, W T}=\int_{-\infty}^{+\infty} F_{\sigma, A, \tau, W T}^{(a i r+t i n) / 2}(\sigma) f_{\sigma, \tau}(\sigma) d \sigma
$$

gives the probability of failure in one year. The parameters defining the action are made to vary until the target values of failure probability in Table 2 is achieved.

At this point, the maximum stress acting in the glass $\sigma_{\max , \tau, d}$ is evaluated via a structural analysis, i.e,

$$
\sigma_{\max , \tau, d}=S\left(\gamma_{f} Q\right)
$$

where we define $S\left(\gamma_{q} Q\right)$ as the stress induced by the action $Q$ of characteristic duration time $\tau$, considered as a deterministic value, multiplied by the partial safety factor $\gamma_{f}$.

The verification formula in the semiprobabilistic approach of level I is of the form

$$
\sigma_{\max , \tau, d}-\sigma_{0, d} \leq \frac{K_{\bmod } \lambda_{A, \tau, W T}\left(f_{g, k}-\sigma_{0, k}\right)}{R_{M} \gamma_{m}}
$$

where $\gamma_{m}$ represents the material partial safety factor and $k_{m o d}$ of (A.5) synthetically takes into account the phenomenon of static fatigue (appendix A). The coefficient $\lambda_{A, \tau, W T}$ accounts for the effects of size and type of stress, while $R_{M}$ provides the correlation with the various classes of consequence. Moreover, $f_{g, k}=45 \mathrm{MPa}$ represents the reference value for the characteristic strength of glass, assumed to refer to the ideal conditions of an equibiaxial 
stress state $\left(K_{W T}=1\right)$ acting on a unitary area $\left(A_{u}=1 \mathrm{~m}^{2}\right)$, and $\sigma_{0, k}$ represents the reference characteristic value of the lower bound for glass strength.

The design value $\sigma_{0, d}$ of the location parameter is taken of the form

$$
\sigma_{0, d}=\frac{K_{m o d} \lambda_{A, \tau, W T} \sigma_{0, k}}{R_{M} \gamma_{m}}
$$

This implies a noteworthy simplification in the verification formula, i.e.,

$$
\sigma_{\max , \tau, d} \leq \frac{K_{\text {mod }} \lambda_{A, \tau, W T} f_{g, k}}{R_{M} \gamma_{m}}
$$

The value $f_{g, k}=45 \mathrm{MPa}$ can be considered representative of the $5 \%$ fractile on the glass strength measured on the air-side under standard reference conditions (uniform equiabiaxial stress on the unitary area) [4].

Assuming the equal probability of failure for a glass plate subjected to a generic stress state acting on the area $A$ and another one under an equibiaxial stress-state acting on a unitary area $\left(A_{u}=1 \mathrm{~m}^{2}\right)$, one can write

$$
1-\frac{1}{2} \sum_{s d=a i r}^{t i n} \exp \left[-\frac{f_{g, k}^{m_{s d}}-\sigma_{0, \tau, s d}^{m_{s d}}}{\eta_{0, \tau, s d}^{m_{s d}}} A_{u}\right]=1-\frac{1}{2} \sum_{s d=a i r}^{t i n} \exp \left[-\frac{K_{W T, s d} \sigma_{m a x}^{m_{s d}}-\sigma_{0, \tau, s d}^{m_{s d}}}{\eta_{0, \tau, s d}^{m_{s d}}} A\right] .
$$

Assuming that the arguments of the exponential functions are infinitesimal of the first order and performing the Taylor's expansion $e^{x}=1+x+o(x)$, after making the substitution $\sigma_{\max } \rightarrow \lambda_{A, \tau, W T} f_{g, k}$, one obtains the condition

$$
\sum_{s d=a i r}^{\text {tin }} \frac{K_{W T, s d}\left(\lambda_{A, \tau, W T} f_{g, k}\right)^{m_{s d}}-\sigma_{0, \tau, s d}^{m_{s d}}}{\eta_{0, \tau, s d}^{m_{s d}}} A=\sum_{s d=a i r}^{\text {tin }} \frac{f_{g, k}^{m_{s d}}-\sigma_{0, \tau, s d}^{m_{s d}}}{\eta_{0, \tau, s d}^{m_{s d}}} A_{u}
$$

An approximate solution for this condition can be obtained by separately evaluating the fracture probability for $s d=$ air and $s d=t i n$, that is 


$$
\frac{K_{W T, s d}\left(\lambda_{A, \tau, W T}^{s d} f_{g, k}\right)^{m_{s d}}-\sigma_{0, \tau, s d}^{m_{s d}}}{\eta_{0, \tau, s d}^{m_{s d}}} A=\frac{f_{g, k}^{m_{s d}}-\sigma_{0, \tau, s d}^{m_{s d}}}{\eta_{0, \tau, s d}^{m_{s d}}} A_{u}
$$

which provides

$$
\lambda_{A, \tau, W T}^{s d}=\frac{1}{f_{g, k}}\left[\frac{f_{g, k}^{m_{s d}} A_{u}+\sigma_{0, \tau, s d}\left(A-A_{u}\right)}{K_{W T, s d} A}\right]^{1 / m_{s d}}, \text { sd }=\text { air }, \text { tin }
$$

Therefore, we will write

$$
\lambda_{A, \tau, \sigma_{\max }}=\lambda_{A, \tau, \sigma_{\max }}^{(a i r+t i n) / 2}=\frac{1}{2}\left[\lambda_{A, \tau, \sigma_{\max }}^{a i r}+\lambda_{A, \tau, \sigma_{\max }}^{t i n}\right] .
$$

This is the final expression that allows the rescaling of the design values in the verification formulas to take into account the effects of size and state of stress.

\subsection{Calibration procedure based on the extended Weibull (EXW) statistics}

Following (2.4), the extended Weibull distribution reads

$$
P_{f, W E}=1-\frac{\nu \exp \left[-K_{W E} A\left(\frac{\sigma_{\max }}{\eta_{0}}\right)^{m}\right]}{1-(1-\nu) \exp \left[-K_{W E} A\left(\frac{\sigma_{\max }}{\eta_{0}}\right)^{m}\right]},
$$

where $\sigma_{\max }$ is the maximum tensile stress, $m$ and $\eta_{0}$ represent the shape and the scale parameters, respectively, while $\nu$ is the third parameter by Marshall \& Olkin [9].

Once the data of the measured strength population has been rescaled to account for the static fatigue phenomenon for loads constantly applied for the time $\tau$, the three parameters characterizing the air and the tin side have been estimated by the graphical method proposed by Zhang and Xie [15]. The value of the parameters defining the distribution for the considered case studies are recorded in Table 4. In elaborating the experimental data, it has been assumed as in Section 3.3 that the effective area is the one associated with a value of the maximum tensile stress equal to $100 \mathrm{MPa}$. 
Table 4: Estimated parameters for the extended Weibull distribution for the wind (two values) and the snow loads. For the test configuration of EN 1288-2 [8]: $K_{W E, a i r}=$ $0.3, K_{W E, \text { tin }}=0.17$.

\begin{tabular}{cccc}
\hline Case study & $m$ & $\begin{array}{c}\eta_{0, \tau} \\
{[\mathrm{MPa}]}\end{array}$ & $\nu_{\tau}$ \\
\hline AIR SIDE & & & \\
Wind, 10 min & 7.9 & 120 & 0.003 \\
Wind, 3 sec & 7.9 & 180 & 0.001 \\
Snow, 1 m & 7.9 & 75 & 0.002 \\
TIN SIDE & & & \\
Wind, 10 min & 12 & 74 & 0.001 \\
Wind, 3 sec & 12 & 100 & 0.002 \\
Snow, 1 m & 12 & 45 & 0.001 \\
\hline
\end{tabular}

Thus, assuming again that the probability of having either the tin side or the air side under tensile stress is the same, equation (3.14) becomes

$$
F_{\sigma, A, \tau, W E}^{(a i r+t i n) / 2}=1-\frac{1}{2} \sum_{s d=a i r}^{\operatorname{tin}}\left[\frac{\nu_{\tau, s d} \exp \left[-K_{W E, s d}\left(\frac{\sigma_{\max }}{\eta_{0, \tau, s d}}\right)^{m_{s d}}\right]}{1-\left(1-\nu_{\tau, s d}\right) \exp \left[-K_{W E, s d}\left(\frac{\sigma_{\max }}{\eta_{0, \tau, s d}}\right)^{m_{s d}}\right]}\right]
$$

The probability of failure in one year then reads

$$
P_{f, 1 y, W E}=\int_{-\infty}^{+\infty} F_{\sigma, A, \tau, W E}^{(a i r+t i n) / 2}(\sigma) f_{\sigma, \tau}(\sigma) d \sigma
$$

The stress induced by the considered action $Q$ of characteristic duration time $\tau$ multiplied by the action partial safety factor $\gamma_{f}$ is given again by equation (3.5). On the other hand, the verification formula takes the simple expression 


$$
\sigma_{m a x, \tau, d} \leq \frac{K_{\text {mod }} \lambda_{A, \tau, W E} f_{g, k}}{R_{M} \gamma_{m}}
$$

where the terms have the same meaning of equation (3.6).

In order to evaluate $\lambda_{A, \tau, W E}$, assuming equal failure probability with standard conditions (unitary area under uniformly distributed loads), one finds

$$
\begin{aligned}
& 1-\frac{1}{2} \sum_{\text {sd=air }}^{\text {tin }}\left[\frac{\nu_{\tau, s d} \exp \left[-K_{W E, \tau, s d} A\left(\frac{\lambda_{A, \tau, W E} f_{g, k}}{\eta_{0, \tau, s d}}\right)^{m_{s d}}\right]}{1-\left(1-\nu_{\tau, s d}\right) \exp \left[-K_{W E, \tau, s d} A\left(\frac{\lambda_{A, \tau, W E} f_{g, k}}{\eta_{0, \tau, s d}}\right)^{m_{s d}}\right]}\right] \\
= & 1-\frac{1}{2} \sum_{\text {sd=air }}^{\text {tin }}\left[\frac{\nu_{\tau, s d} \exp \left[-A_{u}\left(\frac{f_{g, k}}{\eta_{0, \tau, s d}}\right)^{m_{s d}}\right]}{1-\left(1-\nu_{\tau, s d}\right) \exp \left[-A_{u}\left(\frac{f_{g, k}}{\eta_{0, \tau, s d}}\right)^{m_{s d}}\right]}\right]
\end{aligned}
$$

where $f_{g, k}$ is again the reference characteristic strength under standard conditions, and $\lambda_{A, \tau, W E} f_{g, k}=\sigma_{\max }$. To approximately solve this equation, one can again separately evaluate the fracture probability of the air $(s d=a i r)$ and tin $(s d=t i n)$ surfaces, to obtain

$$
\frac{\nu_{\tau, s d} \exp \left[-K_{W E, \tau, s d} A\left(\frac{\lambda_{A, \tau, W E}^{s d} f_{g, k}}{\eta_{0, \tau, s d}}\right)^{m_{s d}}\right]}{1-\left(1-\nu_{\tau, s d}\right) \exp \left[-K_{W E, \tau, s d} A\left(\frac{\lambda_{A, \tau, W E}^{s d} f_{g, k}}{\eta_{0, \tau, s d}}\right)^{m_{s d}}\right]}=\frac{\nu_{\tau, s d} \exp \left[-A_{u}\left(\frac{f_{g, k}}{\eta_{0, \tau, s d}}\right)^{m_{s d}}\right]}{1-\left(1-\nu_{\tau, s d}\right) \exp \left[-A_{u}\left(\frac{f_{g, k}}{\eta_{0, \tau, s d}}\right)^{m_{s d}}\right]},
$$

which implies

$$
\exp \left[-K_{W E, \tau, s d} A\left(\frac{\lambda_{A, \tau, W E}^{s d} f_{g, k}}{\eta_{0, \tau, s d}}\right)^{m_{s d}}\right]=\exp \left[-A_{u}\left(\frac{f_{g, k}}{\eta_{0, \tau, s d}}\right)^{m_{s d}}\right] .
$$

Finally, one obtains

$$
\lambda_{A, \tau, W E}^{a i r}=\left(\frac{A_{u}}{K_{W E, \tau, a i r} A}\right)^{1 / m_{a i r}}, \quad \lambda_{A, \tau, W E}^{t i n}=\left(\frac{A_{u}}{K_{W E, \tau, t i n} A}\right)^{1 / m_{t i n}} .
$$


Then, analogously to (3.13), it will be assumed again

$$
\lambda_{A, \tau, W E}=\lambda_{A, \tau, W E}^{(a i r+t i n) / 2}=\frac{1}{2}\left[\lambda_{A, \tau, W E}^{a i r}+\lambda_{A, \tau, W E}^{t i n}\right] .
$$

in order to evaluate the rescaling of the design values in the verification formulas.

\section{Calibration of partial material factors}

The procedures outlined in Sections 3.3 and 3.4 are now applied to calibrate the material partial safety factors.

\subsection{Wind load}

The procedure is similar to that used by Badalassi et al. [6], but differences arise as a result of the diverse statistical models. As indicated in Section 3.2, consider a simply supported rettangular plate, $3 \times 3 \mathrm{~m}^{2}$ and $8 \mathrm{~mm}$ thick, under the effects of a uniform wind pressure $p_{w}$. The probabilistic model for the wind pressure is that suggested in the standard EN 1991 [16] and reported in Appendix B. Because of the effects of the static fatigue, two characteristic values for $p_{w}$, corresponding to averaged measures on $t=10 \mathrm{~min}$ and $t=3 \mathrm{sec}$, have been considered. The correlation between the maximum tensile stress in the plate and the wind pressure, i.e., $p_{w}=S_{p}(\sigma)$, can be calculated via FEM analysis, so to obtain from equation (B.5) the cumulative distribution function of the maximum stress in the plate consequent to the maximum annual wind pressure, which reads

$$
F_{\sigma, p r, t}(\sigma)=\exp \left[-\exp \left(\frac{1}{0.2}-\frac{2 S_{p}(\sigma)}{\rho c_{e, t} c_{p} c_{d} 0.20 .75^{2} v_{b, 50}^{2}}\right)\right],
$$

where all the coefficients have been introduced in Appendix B. Recall that the difference between the cases $t=10 \mathrm{~min}$ and $t=3 \mathrm{sec}$ is contained in the exposure factor $c_{e, t}$ as per

equation (B.4). The probability density function $f_{\sigma, p r, t}$ is obtained by deriving (4.1) with respect to $\sigma$, i.e., 
$f_{\sigma, p r, t}(\sigma)=F_{\sigma, p r, t}(\sigma) \exp \left(\frac{1}{0.2}-\frac{2 S_{p}(\sigma)}{\rho c_{e, t} c_{p} c_{d} 0.20 .75^{2} v_{b, 50}^{2}}\right) \frac{2}{\rho c_{e, t} c_{p} c_{d} 0.20 .75^{2} v_{b, 50}^{2}} \frac{d}{d x} S_{p}(\sigma)$.

Material strength has been interpreted either with the LTW or the EXW distributions, whose parameters are recalled in tables 3 and 4 for the data recorded in the technical document by CEN/TC129/WG8 [7], re-scaled to take into account the effects of static fatigue for the reference duration of the applied loads.

For the plate of area $A=3 \times 3 \mathrm{~m}^{2}$, the effective area has been evaluated via FEM analyses. The plate has been divided into finite elements of area $\Delta A_{i}=50 \times 50 \mathrm{~mm}^{2}$ and the principal stresses $\sigma_{1}$ and $\sigma_{2}$ estimated at the center of each element. Then, for both the cases $s d=$ air and $s d=t i n$, the equivalent stress $\sigma_{e q, W T, s d, i}$ in the $i$ - th element is evaluated with a formula analogous to (2.2). Then, from (2.3), the effective area $K_{W T, s d} A$ can be calculated as

$$
K_{W T, s d} A=\frac{\sum_{i=1}^{N} \sigma_{e q, W T, s d, i}^{m_{s d}} \Delta A_{i}}{\sigma_{m a x}^{m_{s d}}}
$$

For the extended Weibull statistics, one can obtain an analogous formula for the effective area $K_{W E, s d} A$. Observe that, if non-linear effects are neglected, such expressions depend on plate geometry, constraints and type of loading, but not upon the amount of loading. This assumption provides noteworthy simplifications, it is in general quite accurate. However, here the value is calculated taking into account non linear effects, for a maximum stress of the same order of that used in the calibration procedure. For the case at hand one obtains $K_{W T, a i r}=0.095, K_{W T, t i n}=0.1, K_{W E, a i r}=0.01$ and $K_{W E, t i n}=0.003$.

In order to achieve an optimal design for the selected plate, we can modify the wind pressure $p_{w}$ by artificially changing the location where the structure is set. For example and without loss of generality, the design height above ground $z_{d}$ for both the cases $t=10$ min and $t=3$ sec, so to modify $c_{e, t}$ in (4.2) as per (B.4). The optimal heights are those giving in the convolution integrals (3.4) and (3.16) the target probability of failure of Table 2. 
In the semiprobabilistic method of level I, the design wind pressure is given by (B.3) and must be multiplied by the partial coefficient of the actions $\gamma_{f}=1.5$. The maximum stress is obtained as $\sigma_{\max , d, \tau}=S\left(\gamma_{q} p_{w, d, \tau}\right)$ with FEM analysis. The calibration of the partial material factors then comes from requiring the equal sign in the verification formulae (3.8), for the LTW, and (3.17), for the EXW distributions. Therefore, one finds

$$
\gamma_{m}=\frac{K_{\bmod , \tau} \lambda_{A, \tau, \sigma_{\max }}^{(a i r+t i n)} f_{g, k}}{\sigma_{\max , \tau, d} R_{M}}
$$

where, from (A.5), $k_{\text {mod }}=0.6652$ for $\tau=10$ min and $k_{\text {mod }}=0.9203$ for $\tau=3$ sec.

The values of $\lambda_{A, \tau, W T}^{(a i r+t i n) / 2}$ are estimated through (3.12). Since the variation of the location

parameters only marginally affects the corresponding value, we have set $\lambda_{A, \tau, W T}^{(a i r+t i n) / 2} \simeq 1.034$ for both $\tau=10 \mathrm{~min}$ and $\tau=3 \mathrm{sec}$. On the other hand, $\lambda_{A, \tau, W E}^{(a i r+t i n) / 2}$ is given by $(3.21)$, which provides $\lambda_{A, \tau, W E}^{(a i r+t i n) / 2} \simeq 1.3538$.

Passing to the coefficient $R_{M}$, this is unitary by definition for CC2 elements. The value of $\gamma_{m}$ in (4.4) is the one that corresponds to verification for the CC2 probability of Table 2, with $R_{M}=1$. Once such value has been determined, the other cases of CC1 and CC3 are analyzed and, from (4.4), the corresponding values of $R_{M}$ are determined.

The results of the analysis are summarized in Table 5. Observe that the LTW provides much lower values for $\gamma_{m}$ than the EXW distribution. This despite the fact that for the LTW we have considered the effects of the natural material degradation through the coefficient $\omega$, as explained in Section 2.4, whereas this has been neglected in the EXW approach.

\subsection{Snow load}

Next the plate is subjected to an uniformly distributed snow load $q_{s}$. With FEM analysis one can again find the relationship $q_{s}=S_{q}\left(\sigma_{\max }\right)$ and determine the cumulative distribution of the maximum stress in the plate for the snow loads in one year from (B.9), to obtain 


$$
F_{\sigma, q s, t}(\sigma)=\exp \left[-\exp \left[\left(1-\frac{S_{q}(\sigma)}{q_{s k} \mu_{i} C_{E} C_{t}}(1+2.5923 V)\right) \frac{\pi}{V \sqrt{6}}-0.57722\right]\right]
$$

Differentiating with respect to $\sigma$, one finds the probability density function

$$
\begin{aligned}
f_{\sigma, q s, t}(\sigma) & =F_{\sigma, q s, t}(\sigma) \exp \left[\left(1-\frac{S_{q}(\sigma)}{q_{s k} \mu_{i} C_{E} C_{t}}(1+2.5923 V)\right) \frac{\pi}{V \sqrt{6}}\right. \\
& -0.57722] \frac{\pi}{V \sqrt{6}} \frac{1+2.5923 V}{q_{s k} \mu_{i} C_{E} C_{t}} \frac{d}{d x} S_{q}(\sigma),
\end{aligned}
$$

where all the coefficients have been defined in Appendix B.

To achieve the optimal design with the probabilistic approach, the coefficient of variation of snow loads has been assumed to be $V=0.2$, while the height a.s.l. is varied until the value of the convolution integrals is equal to the target value of the probability of failure. Then, the design load $q_{s, d, \tau}$ is obtained and a deterministic FEM calculation gives the maximum stress $\sigma_{\max , d, \tau}=S\left(\gamma_{f} q_{s, d, \tau}\right)$, with $\gamma_{f}=1.5$. The partial material factors are then calibrated according (4.4). For the case at hand for which $\tau=1$ month, the value of $K_{\text {mod, } \tau}$ is 0.4044

from (A.5), whereas $\lambda_{A, \tau, W T}^{(a i r+t i n) / 2}$ and $\lambda_{A, \tau, W E}^{(a i r+t i n) / 2}$ have the same values as the case of wind load.

Results are shown in Table 5. As in the case of wind, the LTW gives less restrictive coefficients than the EXW approach, although in the second case the effects of natural ageing or glass are neglected.

\subsection{Comparison with previous studies}

An extensive analysis for the calibration of material partial safety factors has been proposed [6], but there are substantial differences with respect to the present derivation. First of all, the considered statistics is a two-parameter Weibull distribution of the type (2.1). Secondly, the shape parameter $m$ and the scale parameter $\eta_{0}$ of the statistics were derived from a 
Table 5: Values of the product $\gamma_{m} R_{M}\left(R_{M}=1\right.$ for CC2), calibrated according to the left-truncated (LTW) and the extended (EXW) Weibull distributions.

\begin{tabular}{ccccccc}
\hline Load case & CC1 (LTW) & CC1 (EXW) & CC2 (LTW) & CC2 (EXW) & CC3 (LTW) & CC3 (EXW) \\
\hline Wind [10 min] - $\gamma_{m} R_{M}$ & 1.62 & 1.71 & 1.82 & 2.30 & 2.04 & 3.20 \\
Wind [3 sec] $-\gamma_{m} R_{M}$ & 1.61 & 1.77 & 1.81 & 2.39 & 2.04 & 3.36 \\
Snow [1 mon] - $\gamma_{m} R_{M}$ & 1.59 & 1.80 & 1.78 & 2.40 & 1.98 & 3.25 \\
\hline
\end{tabular}

particular experimental campaign [17], which is much more limited than that mentioned in Section 2.1, and conducted with a different experimental set-up. Thirdly, to take into account the phenomenon of static fatigue on the reference time $\tau$, the data were re-scaled according to the expressions

$$
m_{\tau}=\frac{n}{n+1} m, \quad \eta_{0, \tau}=\eta_{0}^{\frac{n+1}{n}}\left(\frac{1}{(n+1) \dot{\sigma} \tau}\right)^{1 / n}
$$

where $n$ is the coefficient of the power law (A.1) and $\dot{\sigma}$ is the experimental stress rate, constant and equal to $\dot{\sigma}=2 \mathrm{MPa} / \mathrm{s}$. The relevant values are reported in Table 6 .

Table 6: Weibull parameters used for the calibration of the material partial safety factors according to the 2-parameter Weibull distribution [6].

\begin{tabular}{cccc}
\hline Load Case & Surface & $m_{\tau}$ & $\begin{array}{c}\eta_{0, \tau} \\
{\left[\mathrm{MPa} \mathrm{mm}^{2 / m}\right]}\end{array}$ \\
\hline \multirow{2}{*}{ Test } & Air & 5.4 & 1096 \\
& Tin & 7.3 & 406 \\
\multirow{2}{*}{ Wind, 3 sec } & Air & 5.1 & 1220 \\
\multirow{2}{*}{ Wind, $10 \mathrm{~min}$} & Tin & 6.9 & 425 \\
& Air & 5.1 & 876 \\
\multirow{2}{*}{ Snow, 1 m } & Tin & 6.9 & 305 \\
& Air & 5.1 & 541 \\
& Tin & 6.9 & 188 \\
\hline
\end{tabular}


The case study used by Badalassi et al. [6] for the calibration is also very different, since it considered a $6 \mathrm{~mm}$ thick, $1 \times 1 \mathrm{~m}^{2}$, simply supported square plate. In order to pass from that case to the $3 \times 3 \mathrm{~m}^{2}$ plate, one may use the coefficient $\lambda_{g A}$ taken from the Italian code [12], which adopts the coefficients obtained in the previous work [6], i.e.,

$$
\lambda_{g A}=\left(\frac{0.24 m^{2}}{k A}\right)^{1 / 7} \quad \text { with } k=0.145
$$

Setting $A=9 \mathrm{~m}^{2}$, one obtains $\lambda_{g A}=0.7851$.

Table 7 reports the values of $\left(\gamma_{M} R_{M}\right) / \lambda_{A}$, calculated by using the values of $\gamma_{m}$ and $R_{M}$ given by Badalassi et al. [6] and setting $\lambda_{g A}=0.7851$. The value corresponding to CC3 has been calculated here for the sake of completeness.

\begin{tabular}{|c|c|c|c|}
\hline Load case & CC1 & $\mathrm{CC} 2$ & $\mathrm{CC} 3$ \\
\hline Wind [10 $\mathrm{min}]-\left(\gamma_{M} R_{M}\right) / \lambda_{g A}$ & 2.11 & 3.09 & 4.89 \\
\hline Wind $[3 \mathrm{sec}]-\left(\gamma_{M} R_{M}\right) / \lambda_{g A}$ & 2.27 & 3.2 & 4.97 \\
\hline Snow $[1 \mathrm{mon}]-\left(\gamma_{M} R_{M}\right) / \lambda_{g A}$ & 2.05 & 3.07 & 4.92 \\
\hline
\end{tabular}

Comparing Tables 7 and 5, it is evident that the 2PW distribution provides much higher values of the partial safety factors than the other ones. With respect to the LTW, the hypothesis that the location parameter is null (no lower bound for glass strength) provides a conservative estimate. But the results may be overly conservative for designs that dictate very low failure probabilities of failure. In fact, whereas for CC1 the values of Tables 5 and 7 are different but still comparable, the gap tends to increase for CC3. The EXW distribution leads to safety factors very close to those from the LTW statistics for CC1 elements; slightly higher for CC2 elements; definitely higher for CC3 elements. In any case, the values remain lower than for the $2 \mathrm{PW}$ case. 
Table 8: Values of $R_{M}$ evaluated according to the 2-parameter $(2 \mathrm{PW})$, the extended (EXW) and the left-truncated (LTW) Weibull distributions.

\begin{tabular}{cccc}
\hline & 2PW & EXW & LTW \\
\hline Wind, 10 min & & & \\
$R_{M}(\mathrm{CC} 1)$ & 0.68 & 0.74 & 0.89 \\
$R_{M}(\mathrm{CC} 3)$ & 1.58 & 1.39 & 1.12 \\
Wind, 3 sec & & & \\
$R_{M}(\mathrm{CC} 1)$ & 0.71 & 0.74 & 0.89 \\
$R_{M}(\mathrm{CC} 3)$ & 1.55 & 1.41 & 1.13 \\
Snow, 1 month & & & \\
$R_{M}(\mathrm{CC} 1)$ & 0.67 & 0.75 & 0.89 \\
$R_{M}(\mathrm{CC} 3)$ & 1.60 & 1.35 & 1.11 \\
\hline
\end{tabular}

The calibrated values of $R_{M}$ are summarized in Table 8 for all the considered cases. Recall from Section 3.2 that the Eurocode EN 1990 [3] provides the multiplicative coefficient $K_{F I}$ for the actions to pass from CC2 to CC1 $\left(K_{F I}=0.9\right)$ or to CC3 $\left(K_{F I}=1.1\right)$, and that for linearly elastic structure such coefficient plays the same role of $R_{M}$. Although this comparison is of limited significance because the statistics assumed to define the values of $K_{F I}$ is not Weibull's, one finds from Table 8 that the left truncated Weibull distribution is the one that gives results in excellent agreement with the procedure suggested by the Eurocode for all building materials.

\section{Discussion and conclusions}

This article has presented a new calibration of partial material factors to be used in the structural design of glass with methods of level I (semiprobabilistic approach). The calibration was obtained from the comparison of the results obtainable with methods of level III (full probabilistic) in paradigmatic case studies. The statistical models for the effects of the applied actions, essentially wind and snow, are consistent with those proposed in the EN 
1991-1-4 [16]. The performance of the structure is given by the maximum allowed probability of failure, which is established in the EN 1990 [3] for three classes of consequences. A proposal is made here to extend such classes to the case of localized glass elements whose failure, although not provoking the collapse of the whole buiding, can have consequences of various levels of severity.

This study complements and develops the results recorded in a previous paper [6], where a similar-in-type procedure was followed, but with substantial differences. First of all, the type of statistical model for glass strength is different. Whereas in the previous work [6] the attention was focused on the two-parameter Weibull (2PW) distribution, here we have used the extended (EXW) and left-trunccated (LTW) generalized Weibull statistics. According to a recent study [5], these are able to interpret the left-hand-side tail of the population much better than the more classical 2PW model, a property that is of crucial importance while dealing with low probabilities of failure likewise those tolerated in construction works. In particular, the LTW prescribes a lower bound for glass strength, a quite delicate hypothesis whose significance has been discussed at length [5]. Proper rescaling of the glass strengths are proposed to account for static fatigue due to subcritical crack propagation and for the effects of size and type of stress. Moreover, the experimental campaigns, from which the parameters defining the statistics have been derived, are quite different. Whereas in the previous study [6] reference is made to the tests performed by the italian "Stazione Sperimentale del Vetro" [17], performed with a ring-on-ring set-up, here we have used the results of the much wider experimental campaign of the working group TC129/WG8 of CEN, performed according to EN 1288-2 [8], and statistically elaborated by the authors of the paper in previous works $[4,5]$.

The data obtained here are summarized in Table 5, and should be compared to those of Table 7, which refers instead to the previous study [6]. The difference is quite striking: the partial factors from the $2 \mathrm{PW}$ may be as much as twice those derived from the LTW, the difference being more evident at the lowest probabilities of failure (class of consequence CC3). The EXW distribution provides values that are intermediate between the aforementioned cases, 
even if for this statistics the effects of aging have been neglected, whereas they have been considered in the LTW case.

There are reasons to repute that the most reliable estimate are those obtainable with the lefttruncated Weibull statistics. On the one hand, the statistical analysis has demonstrated the inability of the 2PW distribution to interpret the left-hand-side of the strength population [4]. On the other hand, the possibility of the existence of the lower bound for glass strength can be justified on the basis of the severe factory production controls used during manufacturing [5]. Indeed the distributions that indicate such lower bound have been proved to be the best in the interpretation of the data corresponding to the lower probabilities. Moreover, experimental results have demonstrated that also severe damaging action, like sandblasting, cannot reduce the strength of glass beyond a certain lower limit. The possibility of such a degradation has been here accounted for by diminishing, on the safe side, the limit strength calculated from experiments.

The results obtained by Badalassi et al. [6] have been considered overly conservative by the building industry and professional designers, because they are not consistent with the coefficients traditionally used on the basis of experience and rules of practice. One can argue that the $2 \mathrm{PW}$ statistics is on the safe side, but its overly conservative nature reduces the competitiveness of glass in construction. Since the idea of a lower bound for glass strength may be difficult to accept, because it is in conflict with the engineering sense according to which nothing can be $100 \%$ safe, the partial factors have been also calibrated using the EXW statistics, which is an "unbounded" distribution giving a good, even though not optimal, interpretation of the tails of the population of strengths [5]. Partial safety factors remain in any case much less than those obtained by using the $2 \mathrm{PW}$ statistics.

It should also be mentioned that the Eurocode EN 1990 [3] provides the coefficient $K_{F I}$, multiplicative of the applied actions, to pass from verifications in class of consequence CC2 to CC3 or CC1. Here, this passage is proposed through the coefficient $R_{M}$ that multiplies not the actions, but the material strengths. This is because of the slenderness of glass structures, for which geometric non linearities may be so important to render preferable 
maintaining the actions unaltered, but the effects of $K_{F I}$ and $R_{M}$ are completely equivalent if the structure is linear elastic. What should be observed is that the variation associated with $K_{F I}$, as suggested in EN 1990 [3], is approximately $10 \%$, and such a value squares very well with the results from the LTW statistics. Higher differences are obtained for the EXW, and even more so with the $2 \mathrm{PW}$ distributions. Although the importance of the comparison is not absolute, since the coefficients $K_{F I}$ have been calibrated in EN 1990 [3] on the basis of different-in-type statistics, this finding confirms the opportunity to comply with general principles of structural design, commonly adopted for all types of materials.

The presented results are certainly not exhaustive, since there are some unsolved matters and still-open problems. First of all, the Weibull parameters for the extended and left truncated Weibull distributions have been here estimated graphically, but a more rigorous analytical method may provide a slightly different estimation of the partial material factors. Moreover, the considered case study is such that the maximum tensile stress acts far from the border, but there are important structural elements, like fins or beams, in which the edge is under maximum tension. The strength of the edge can be quite different from that of the core material due to the damage induced during the cutting process, but the lack of experimental data does not allow for a reliable statistical characterization. A similar consideration applies to surface treatments, as well as to heat or chemically toughened glass. Another critical issues regards the effects of live personnel loads, whose stochastic interpretation is still not definite, to our knowledge.

This study does demonstrate that the values of partial material factors considered so far on the basis of the study of Badalassi et al. [6] are overlyconservative. Industrial competitiveness in the globalized market asks for reliable and safe construction that at the same time comply with the issues of cost-effectiveness, energy savings and reduction of pollutant emission (recall that the embodied energy of glass is comparable with that of steel, and about 15 times greater than for concrete). We hope that new experimental researches will be supported to corroborate the new calibration procedure presented here, in order to permit the adoption of milder design coefficients in current structural standards. 
Acknowledgement. R.B. acknowledges the Thomas and Laura Hsi Professorship and the National Science Foundation under Grant CMMI -1361868. G. P. and G. R. C. acknowledge the support of the Italian Dipartimento della Protezione Civile under project ReLUIS-DPC 2014-2018.

\section{Appendix A. The effective crack growth model}

Brittle failure of glass is governed by surface micro-cracks that propagate catastrophically once the crack opening stress reaches a critical value [18]. The phenomenon can be well interpreted by linear elastic fracture mechanics (LEFM). Crack growth is governed by the Stress Intensity Factor $(\mathrm{SIF}) K_{I}$ in mode I, and instantaneous collapse occurs when the SIF reaches the critical threshold $K_{I c}$ [19], which represents a characteristic material parameter $\left(K_{I c}=0.75 \mathrm{MPa} \mathrm{m} \mathrm{m}^{0.5}\right.$ is usually assumed).

A subtle phenomenon, usually referred to as static fatigue or subcritical crack propagation [1], is the slow growth over time of cracks while their size is far smaller than the critical limit. The speed of subcritical crack growth is traditionally considered a function of the SIF, governed by a power-law of the type [20]

$$
\frac{d c}{d t}=\nu_{0}\left(\frac{K_{I}}{K_{I C}}\right)^{n},
$$

where $\nu_{0}$ and $n$ are parameters that depend upon the thermo-hygrometric conditions and the type of glass [21]. It customary to assume $\nu_{0}=0.0025 \mathrm{~m} / \mathrm{s}$ and $n=16$ for float soda-lime glass to be on the safe side.

The subcritical crack growth occurs when micro-cracks are under a positive crack opening stress for value of the SIF comprised between $K_{I 0}$, a lower bound below which no propagation occurs but that is usually neglected, and $K_{I C}$. Since the SIF for a thumbnail surface crack is $K_{I}=\sigma Y \sqrt{\pi c}$, where $\sigma$ is the crack opening stress, $Y$ is a shape coefficient and $c$ is the 
characteristic size of the $\mathrm{crack}^{2}$, when considering a load history $\sigma=\sigma(t)$ for the crack opening stress, one can write

$$
\int_{c_{i}}^{c_{c}} c^{-n / 2} d c=\int_{0}^{t_{f}} \nu_{0}\left(\frac{\sigma(t) Y \sqrt{\pi}}{K_{I c}}\right)^{n} d t
$$

where $t_{f}$ represents the failure time, when the crack size passes from the initial value $c_{i}$ to the critical value $c_{c}$.

Considering that tests are, as a rule, performed at a constant stress rate $\dot{\sigma}_{\text {test }}$, then $\sigma(t)=$ $\dot{\sigma}_{\text {test }} t$. If $f_{\text {test }}$ is the tensile strength measured at the end of the test, from (A.2) one can find the initial crack size $c_{i}$

$$
c_{i}=\left[\frac{n-2}{2} \frac{\nu_{0}}{n+1}\left(\frac{Y \sqrt{\pi}}{K_{I c}}\right)^{n} \frac{f_{\text {test }}^{n+1}}{\dot{\sigma}_{\text {test }}}+\left(\frac{Y f_{\text {test }} \sqrt{\pi}}{K_{I c}}\right)^{n-2}\right]^{\frac{2}{n-2}},
$$

which indicates a measure of the initial defectiveness of the glass specimen. For practical values of the parameters, the second term within the square brackets is negligible with respect to the first one. Under this assumption, since $c_{i}$ is a given quantity, it turns out that $f_{\text {test }}^{n+1} / \dot{\sigma}_{\text {test }}=R$ is approximately constant, whatever the stress rate during the test.

For design purposes, actions are schematized by constant loads acting on the structure for a characteristic time, representative of their cumulative effect during the whole life-time. Suppose that the stress $\sigma_{\tau}$ is sufficient to provoke rupture if constantly applied for the time $\tau$. Integrating equation (A.2), one can write [6]

$$
\sigma_{\tau}^{n} \tau=\frac{2 /(n-2) c_{i}^{(2-n) / 2}}{\nu_{0}\left(\frac{Y \sqrt{\pi}}{K_{I c}}\right)^{n}}=\frac{1}{n+1} R
$$

From this expression, it is then possible to obtain, for any fixed $\tau$, the stress $\sigma_{\tau}$.

\footnotetext{
${ }^{2}$ Since thumbnails cracks naturally tend to be semicircular, one can identify $c$ with the radius of the crack and assume $Y=2.24 / \pi$.
} 
For design purpose, a more practical way to consider this phenomenon is through the coefficient $k_{m o d}$, which takes the form

$$
k_{\text {mod }}=\frac{\sigma_{\tau}}{f_{\text {ref }}}=\frac{1}{f_{\text {ref }}}\left(\frac{1}{n+1}\right)^{1 / n}\left(\frac{\tau}{R}\right)^{-1 / n}=\left(\frac{1}{n+1}\right)^{1 / n}(R)^{\frac{1}{n(n+1)}}\left(\dot{\sigma}_{r e f}\right)^{\frac{-1}{n+1}}(\tau)^{-1 / n}
$$

Here $f_{r e f}$ is a reference value, which is generally assumed to be the bending strength of a glass plate tested at $\dot{\sigma}_{r e f}=2 \mathrm{MPa} / \mathrm{sec}$. For float glass, by assuming characteristic values for the various parameters, one obtains for $n=16$ the expression $k_{\text {mod }}=0.9759(\tau)^{1 / 16}$ when $\tau$ is measured in seconds.

\section{Appendix B. Probabilistic models for wind loads and snow loads}

Wind actions are usually considered as uniformly distributed pressures. There are various

probabilistic models for wind pressure. According to the Eurocode EN1991-1-4 [16], the reference wind velocity corresponding to a return period $T_{R}$ is of the form

$$
v_{b}\left(T_{R}\right)=\alpha_{R} v_{b, 50}, \quad \alpha_{R}=0.75 \sqrt{1-0.2 \ln \left[-\ln \left(1-\frac{1}{T_{R}}\right)\right]},
$$

where $v_{b, 50}$ is defined as the characteristic wind velocity at $10 \mathrm{~m}$ above ground averaged over 10 minutes on an exposure category II field, determined for a return period of 50 years. From equation (B.1), it is possible to obtain the cumulative distribution function of the maximum averaged wind velocity over 10 minutes recorded in one year $v_{b}$, i.e., the probability that such value is not exceeded in one year, which reads

$$
F\left(v_{b}\right)=\exp \left[-\exp \left(\frac{1}{0.2}-\frac{v_{b}^{2}}{0.20 .75^{2} v_{b, 50}^{2}}\right)\right]
$$

According to EN1991-1-4 [16] the peak pressures, corresponding to the averages over time $t=10 \mathrm{~min}$ and $t=3 \mathrm{sec}$, are indicated by 


$$
p_{w, 10 \mathrm{~min}}=\frac{1}{2} \rho v_{b}^{2} c_{e 1}(z) c_{p} c_{d}, \quad p_{w, 3 s}=\frac{1}{2} \rho v_{b}^{2} c_{e}(z) c_{p} c_{d} .
$$

Here, $c_{d}$ is the dynamic factor, $c_{p}$ is the pressure coefficient, $\rho=1.25 \mathrm{Kg} / \mathrm{m}^{3}$ is the air density, $z$ is the height above ground, whereas $c_{e 1}(z)$ and $c_{e}(z)$ are the exposure factors. These factors, which mark the distinction between the peak pressure corresponding to the averaged time $\mathrm{t}=10$ min or $\mathrm{t}=3 \mathrm{~s}$, are given by

$$
\begin{aligned}
c_{e 1}(z) & =\left(\ln \left(\frac{z}{z_{0}}\right)\right)^{2} k_{r}^{2} c_{t}^{2} \quad \text { with } z=z_{\min } \text { for } z \leq z_{\min }, \\
c_{e}(z) & =k_{r}^{2} c_{t}(z) \ln \left(\frac{z}{z_{0}}\right)\left[\ln \left(\frac{z}{z_{0}}\right) c_{t}(z)+7\right] \quad \text { with } z=z_{\min } \text { for } z \leq z_{\min },
\end{aligned}
$$

where $z_{0}$ and $z_{\min }$ are reference heights, $k_{r}$ is a coefficient that depends upon the field exposure category and $c_{t}(z)$ is the orographic coefficient. Hence, the cumulative distribution function $F\left(p_{w, t}\right)$ takes the form

$$
F\left(p_{w, t}\right)=\exp \left[-\exp \left(\frac{1}{0.2}-\frac{2 p_{w, t}}{\rho c_{e, t} c_{p} c_{d} 0.20 .75^{2} v_{b, 50}^{2}}\right)\right],
$$

with $c_{e, t}=c_{e}$ when $\mathrm{t}=3 \mathrm{~s}$ and $c_{e, t}=c_{e 1}$ when $\mathrm{t}=10 \mathrm{~min}$.

The snow actions are due to the snow deposit on a roof, considered undrifted for uniform flat surfaces. The equation for the snow load referred to a return period of $n$ years $\left(q_{s n}\right)$ is proposed in the standard EN 1991-1-3 [22] through the expression

$$
q_{s n}=q_{s k}\left[\frac{1-V \sqrt{6} / \pi\left[\ln \left(-\ln \left(P_{n}\right)\right)+0.57722\right]}{1+2.5923 V}\right],
$$

where $V$ is the variation coefficient of the series of maximum annual snow loads, $P_{n}$ is the annual probability of exceedance and $q_{s k}$ is the characteristic snow load value on the ground for a 50 year return period. Such value is reported by regulations as a function of the climate zone and the altitude above sea level. From (B.6), one can obtain the value of the annual 
probability of non-exceedance and, hence, the cumulative distribution function for ground snow loads for a reference period of 1 years, which results to be

$$
F_{q s n}(x)=\exp \left[-\exp \left[\left(1-\frac{x}{q_{s k}}(1+2.5923 V)\right) \frac{\pi}{V \sqrt{6}}-0.57722\right]\right] .
$$

By denoting with $C_{E}$ the exposure coefficient, with $C_{t}$ the thermal coefficient, and with $\mu_{i}$ the roof shape coefficient, the snow load on the roof is determined by the expression

$$
q_{s}=\mu_{i} q_{s k} C_{E} C_{t}
$$

Then, by substituting equation (B.8) into (B.7), one obtains the cumulative distribution function of undrifted snow loads on roofs

$$
F_{q s}(x)=\exp \left[-\exp \left[\left(1-\frac{x}{q_{s k} \mu_{i} C_{E} C_{t}}(1+2.5923 V)\right) \frac{\pi}{V \sqrt{6}}-0.57722\right]\right]
$$

The values of the various coefficients can be found in the EN 1991-1-3 [22].

\section{References}

[1] S.M. Wiederhorn and L.H. Bolz. Stress corrosion and static fatigue of glass. J Am Ceram Soc, 53(10):543-548, 1970.

[2] W. Weibull. A statistical distribution function of wide applicability. J. Appl. Mech., 18:293-297, 1951.

[3] CEN-TC250. EN1990:2005 - Eurocode 0 - Basis of structural design. European Standard, 2005.

[4] G. Pisano and G. Royer-Carfagni. The statistical intepretation of the strength of float glass for structural applications. Constr Build Mater, 98:741-756, 2015. 
[5] R. Ballarini, G. Pisano, and G. Royer-Carfagni. Is there a lower bound for glass strength? A statistical approach á la Weibull for structural applications. Submitted, 2016.

[6] Massimo Badalassi, Luigi Biolzi, Gianni Royer-Carfagni, and Walter Salvatore. Safety factors for the structural design of glass. Constr Build Mater, 55:114 - 127, 2014.

[7] CEN/TC129/WG8. An overview of prEN-13474 and the work of CEN/TC129/Wg8 from which it was developed. Internal document, February 2006.

[8] CEN/TC129. EN 1288-2:2001 - Glass in building - Determination of bending strength of glass - Part 2: Coaxial double ring test on flat specimens with large test surface areas. European Standard, 2001.

[9] A.W. Marshall and I. Olkin. A new method for adding a parameter to a family of distributions with application to the exponential and weibull families. Biometrika, 84(3):641-652, 1997.

[10] S. Schula, J. Schneider, M. Vandebroek, and J. Belis. Fracture strength of glass, engineering testing methods and estimation of characteristic values. In Proc COST Action TU0905 Mid-Term Conf Struct Glass, pages 223-234, 2013.

[11] X. Xiao and X. Yan. A numerical analysis for cracks emanating from a surface semispherical cavity in an infinite elastic body by franc3d. Eng Fail Anal, 15:188 - 192, 2008.

[12] CNR-DT/210. Guide for the Design, Construction and Control of Structures made of Glass Structural Elements. National Research Council (CNR), 2013. [In Italian].

[13] ASTM E1300 - 12ae1. Standard practice for determining load resistance of glass in buildings. The American Society for Testing and Materials, 2002. 
[14] G. Pisano and G. Royer-Carfagni. Towards a new standardized configuration for the coaxial double test for float glass. submitted. Preprint available on http://www.ativonline.it/Indexus.htm, I.R.S.A.G., 2016.

[15] T. Zhang and M. Xie. Failure data analysis with extended weibull distribution. Commun Stat B-Simul, 36(3):579-592, 2007.

[16] CEN-TC250. EN1991-1-4:2005 - Eurocode I - actions on structures - Part 1-4: General actions - wind actions. European Standard, 2005.

[17] R. Dall'Igna, A. D'Este, and M. Silvestri. Comments on test methods for the determination of structural glass strenght. In Proc XXV ATIV Conf, Parma (Italy), pages 5-13, 2010.

[18] Matteo Ciccotti. Stress-corrosion mechanisms in silicate glasses. J. Phys. D: Appl. Phys., 42(21):214006.1-214006.8, 2009.

[19] SM Wiederhorn. Fracture surface energy of glass. J. Am. Ceram. Soc., 52(2):99-105, 1969.

[20] A. G. Evans. A general approach for the statistical analysis of multiaxial fracture. J. Am. Ceram. Soc., 61:302-308, 1978.

[21] Sheldon M Wiederhorn, Stephen W Freiman, ER Fuller Jr, and CJ Simmons. Effects of water and other dielectrics on crack growth. J. Mater. Sci., 17(12):3460-3478, 1982.

[22] CEN-TC250. EN1991-1-3:2003 - Eurocode I - actions on structures - Part 1-3: General actions - snow loads. European Standard, 2003. 